\title{
Français inclusif : conceptualisation et analyse linguistique
}

\author{
Alpheratz \\ EDP Paris Sorbonne Université, Ecole doctorale V Concepts et langages \\ ED 0433 \\ contact@alpheratz.fr
}

Résumé. Cet article synthétise la conceptualisation du «français inclusif » comme ensemble de variations du français standard fondées sur le genre et comme variété en usage dans plusieurs groupes sociaux ayant pour point commun une conscience et/ou une politique de genre. Propre au $\mathrm{XXI}^{\mathrm{e}}$ siècle, ce phénomène produit des observables que la recherche linguistique peut se donner comme objet d'étude. Sa description et sa conceptualisation nous obligeaient à définir le cadre philosophique et la communauté épistémique ayant présidé à son émergence mais aussi à élaborer les outils et les termes métalinguistiques servant à son analyse. Ainsi, «la variation diaéthique, l'épicénisation, l'écriture inclusive» ou «double flexion partielle » et «l'hyperonymisation de genre » sont des processus mais également de nouvelles ressources linguistiques permettant d'étudier ces nouveaux usages. Certains énoncés peuvent relever d'une catégorie grammaticale disparue en français : le genre neutre. Nous verrons quelles sont les perspectives de sa réactivation et en quoi cette catégorie grammaticale permet peut-être de répondre à un besoin que le français standard échoue à satisfaire. Enfin, nous présenterons une introduction à l'expérimentation linguistique du genre neutre que nous menons, et grâce à laquelle se trouvent les instances de validation et de réfutation de l'hypothèse de son existence et de son utilité.

\footnotetext{
Abstract. Inclusive French : Conceptualization And Linguistic Analysis. This research paper summarises the conceptualization of "inclusive French" as a set of variations of standard French based on gender and used by several social groups which have in common a gender consciousness and/or politics. Specific to the 21st Century, this phenomenon has been producing observables that linguistics can see as an object of study. Its description and its conceptualization forced us to define the philosophical context and the epistemic community that contributed to its emergence and to design the concepts and the metalinguistic terms for its analysis. "The diaethical variation, the epicenization, the inclusive writing" and "the gender hyperonymization" are linguistic processes and resources that allow to study these new usages. Some of them can fall under a grammatical category which has disappeared in French : the neutral gender. We will see what are the prospects of its reactivation and how, perhaps, this grammatical category can satisfy a need that standard French doesn't manage to fulfil. Finally, we will introduce our linguistic experimentation on the neutral gender that will allow us to validate or invalidate the hypothesis of its existence and its usefulness.
} 


\section{INTRODUCTION}

«Variation diaéthique, épicénisation, hyperonymisation, écriture inclusive » ou « double flexion partielle », etc. Le troisième millénaire voit émerger de nouveaux outils et ressources pour l'analyse linguistique - termes et concepts - dans le cadre de l'étude d'une variété du français standard que nous nommons « le français inclusif ». Nous proposons ici de définir ce concept, de rappeler le cadre qui l'a vu naître et d'examiner ces nouveaux termes métalinguistiques qui nous semblent nécessaires à son analyse.

Depuis les années 2000, les dérivations suffixales, à partir de la base inclus-, se multiplient dans les usages :

Ex. outils réalisés par la Fédération, ou conçus par d'autres partenaires sensibilisés à la question du handicap et de l'inclusion dans la société.

(apajh.org/reflexion-action/enfance-jeunesse/actualites/journee internationale des personnes handicapees $12 / 11 / 2012$ )

Ex. Quelle école pour une société inclusive?

(inshea.fr/Actualités éducation inclusive)

Ex. beaucoup de féministes se présentent comme intersectionnelles pour se revendiquer d'une certaine forme de féminisme, caractérisée par l'inclusivité.

(Blog Ça fait genre/Des questions?)

En 2004, dans son essai de philosophie politique Défaire le genre, la philosophe et théoricienne américaine du genre social Judith Butler s'interroge sur la nécessité d'une " resignification " (à la fois réappropriation et "renomination ») des concepts et des catégories ayant pour objectif une politique d'intégration de catégories sociales minorisées. Pour elle, cette resignification seule ne suffit pas, elle doit être « inclusive » :

« Nous devons considérer que nos conventions préétablies quant à ce qui est humain, ce qui est universel et ce que sont la signification et la substance des politiques internationales ne suffisent pas. Pour une transformation démocratique radicale, il faut que nous sachions que nos catégories fondamentales peuvent et doivent être étendues pour devenir plus inclusives et plus responsables face à l'ensemble de l'éventail des populations culturelles. » (Butler 2004 : 253, souligné par nous-même.)

Si le propos relève ici de la philosophie politique, nous ne pouvons faire l'économie de sa compréhension pour comprendre pourquoi nous reprenons l'adjectif « inclusif » à notre compte afin de l'intégrer au lexique métalinguistique.

Dans la philosophie de Butler, le principe d'inclusivité est une stratégie politique visant à inclure les sujets « sensibles » au sens de "précaires, vulnérables, minorisés » et à visibiliser une idéologie dominante au sens de «normative, prescriptive, excluante » dont la prétention est de détenir la vérité et de représenter une pensée universelle.

Le français inclusif relève du même principe d'inclusivité. Cette variété du français standard applique des processus langagiers dont le point commun est d'inclure dans 
l'ontogénèse (pour reprendre la terminologie guillaumienne) les genres sociaux minorisés par l'emploi générique du genre grammatical masculin - lequel prétend exprimer une pensée universelle - et de visibiliser à la fois le principe grammatical excluant (le genre masculin en emploi générique) et les représentations symboliques ou sociales que cet emploi n'exprime pas.

Ces processus langagiers s'observent principalement dans l'expression de la personne, mais également dans celle de réalités agenres (notamment dans les structures impersonnelles), des animaux et des concepts. Ils relèvent d'une conduite raisonnée - donc d'une politique - qui prend naissance dans la pensée du locutaire ${ }^{i}$ et active une conscience de genre dans celle de l'allocutaire. L'inclusivité, en métalinguistique, décrit donc des faits intralinguistiques (double flexion totale ou partielle, insertion de signes de ponctuations, néologie, réactivation de mots ou de régularités anciennement attestées, etc.) :

Ex. Il apparaît que les élèves ont toujours plus confiance en elles et en eux pour les professions qui sont stéréotypiquement de leur genre (double flexion totale)

(ONISEP $2014: 11$ )

Ex. Quand on partage le texte de quelqu'un-e, on L'ENTOURE de GUILLEMETS (double flexion partielle)

(Profil facebook de Flo Morandet, 30/08/2017)

Ex. Aime ton enfant tel qu'iel est ! (Néologie)

(Profil facebook de Clémence Zamora-Cruz, 04/09/2017)

Ex. Au lieu d'ancrer ainsi la domination dans la langue, la règle de proximité amène à écrire : "Les hommes et les femmes sont belles » (Réactivation de l'accord de proximité)

(Editions iXe, dans Viennot $2014: 3$ )

Parce qu'il qualifie des faits de langue, l'adjectif « inclusif » nous permet de rester sur le terrain de la description scientifique, alors qu' " égalitaire », par exemple, par sa signification sociale dépendant du positionnement idéologique de qui l'utilise, ne nous semble pas doté d'un niveau de spécialisation linguistique suffisamment élevé pour devenir un terme de métalangue.

Le « français inclusif » peut donc se définir comme l'ensemble des processus langagiers qui s'écartent du français standard, fondés en général sur la notion de genre, et en particulier sur le rejet d'une hiérarchie entre les représentations symboliques et sociales associées aux genres grammaticaux, ces variations ayant pour objectif d'inclure et de visibiliser tous les genres dans la langue comme dans la pensée (Alpheratz 2018).

Le français inclusif est un idiome, selon la définition que Franck Neveu donne de ce dernier terme (2004: 156) : il désigne des pratiques linguistiques qui sont considérées comme spécifiques par rapport à la langue à laquelle elles se rattachent. Ce n'est pas un dialecte si nous considérons comme André Thibault (2018) qu'un dialecte se rattache strictement à la notion d'espace. Peut-être que les attestations de plus en plus nombreuses $\mathrm{du}$ français inclusif et l'apparition de guides de rédaction et de grammaires qui le normativisent inscrivent cette variété dans la catégorie des langues par élaboration (Kloss $1987^{\mathrm{ii}}$, cité par Thibault 2018). En effet, le français inclusif répond à tous les critères donnés par Kloss, bien que ce phénomène soit en émergence, et sans que tous ses processus langagiers aient le même succès dans l'usage (certains, comme la féminisation des noms de métier, sont courants, d'autres, comme le genre neutre, donnent lieu à des hapax) : 
1) « être utilisée comme langue de la presse ;

2) être utilisée comme langue dans laquelle sont traduits des textes religieux ou idéologiques ;

3) être utilisée dans des situations formelles d'élocution [...];

4) être utilisée dans des textes écrits bassement utilitaires, donc autres que des textes littéraires [...] ». (critères de Kloss donnés par Thibault 2018).

Cette variété ne comporte pas de diglossie, elle présente même la particularité d'être valorisée. Cette valorisation est due à la catégorie socio-professionnelle de ses locutaires qui appartiennent (en partie) au monde politique, du journalisme, de l'entreprise, de l'art, de la recherche linguistique et des institutions (Alpheratz 2018). Elle l'est également par la glottopolitique qui cherche à la promouvoir, notamment par le biais de guides de rédaction (Becquer et al. 1999, Guénette \& Vachon-L'Heureux 2006, ONISEP 2014, HCE 2016).

Cette variété n'est pas circonscrite à une zone géographique ni à une seule catégorie sociale. Tous les individus ou les groupes qui l'emploient (en totalité ou partie) ont ce point commun : une conscience relative à la notion de genre, point commun sur lequel nous fondons "la variation diaéthique », autre concept et terme métalinguistique que nous pensons devoir faire figurer au glossaire de notre discipline pour étudier le français inclusif et que nous définissons ci-après.

\section{Cadre historique et communauté épistémique}

Le français inclusif est un phénomène qui est d'abord lié aux situations d'énonciation propres au $\mathrm{XXI}^{\mathrm{e}}$ siècle, lequel se distingue par cette (r)évolution technologique majeure que représente le passage de l'écrit au numérique, majeure ne serait-ce que pour la linguistique, dont il oblige à redéfinir le champ d'analyse, compte tenu de la massification des données, du bouleversement du statut de l'auctorialité et de l'accès immédiat au savoir (Schmitt 2015, Neeman 2012, Serres 2011).

La pratique de l'écrit sur internet diffère également de son pendant sur papier en créant ces nouveaux espaces textuels que sont les publications individuelles, très différentes selon qu'elles prennent place dans le cadre d'un commentaire ou d'un livre de e-littérature, ou encore selon qu'elles sont publiées sur un profil facebook, un compte twitter, un blog ou un site. Ces espaces ont libéré une créativité néologique dans les usages qui nous semble avoir contribué à la naissance du français inclusif :

Ex. Beaucoup de réflexions ont notamment été menées avec l'autrim de ce blog.

(Blog Unique en son genre/Accueil, 26/01/2016)

Ex. Il a été créé par des travailleurSEs du sexe lors des Assises européennes de la prostitution

(strass-syndicat.org/Qui sommes-nous?)

Ex. On peut également écrire « celleux » ou « ceulles » pour " celles et ceux "

(Lady Dylan, madmoizelle.com/Culture/Guide du langage non sexiste, 12/06/2012)

Autre paramètre qui nous semble avoir présidé à cette naissance : la déconstruction des systèmes de pensée dominants par les sciences humaines à partir de la seconde moitié du $\mathrm{XX}^{\mathrm{e}}$ siècle. Nous pensons plus précisément à la remise en cause du sujet cartésien par les théories de son évanouissement en psychanalyse (Lacan, 1966), du structuralisme et de la 
déconstruction en philosophie (Althusser 1964, Derrida 1966, Foucault 1966 et 1975), de la domination masculine en anthropologie et sociologie (Delphy 1975, Guillemin 1978, Héritier 1996), du genre comme construction sociale dans les études éponymes (Kosofsky Sedgwick 1990, Butler 1990 et 2004), ou encore de l'européocentrisme dans les études post coloniales (Saïd 1980, Bhabha 2007).

A ce vaste mouvement analysant les mécanismes de pouvoir à l'œuvre dans les rapports humains viennent s'ajouter en linguistique les travaux explorant l'interdépendance de la pensée et du langage, et la capacité du signe à créer de la réalité, que ce soit par sa performativité (Austin 1962), sa construction sociale (Sapir 1949, Whorf 1956, Searle 1995), son structuralisme (Propp 1928, Genette 1966, Barthes 1971), son analogie ou son iconicité (Jacobson 1956 et 1965, Fischer \& Nänny 1999, Monneret 2011, Nobile 2014).

L'intérêt que constituent les rapports isomorphes entre genre grammatical et genre social se diffractent aussi, et plus précisément, à travers les études de la langue française, que ce soit en sociolinguistique (Yaguello 1978), en sémantique (Michard 2012, Michel 2016), en morphosyntaxe (Labrosse 1996, Dressler \& Kilani Schoch 2014, Elmiger 2017), en lexicologie (Khaznadar 1989) ou en histoire de la langue (Viennot 2014).

Enfin, la littérature, avec des œuvres du troisième millénaire fondées en partie sur le bouleversement du traitement grammatical des genres (Vonarburg 1999, La Baronne 2007, Rozenfeld 2014, Alpheratz 2015, D. 2016) voit l'émergence de styles qui, dans le dialogue qu'ils instituent entre autaire $^{\text {iii }}$ et lectaire ${ }^{\text {iv }}$, selon une perspective bakthinienne, contribuent à l'émergence d'une conscience de la hiérarchisation androcentrique d'un système de valeurs (sémantiques) par la grammaire et l'intériorisation par la communauté linguistique $\mathrm{du}$ français standard de processus langagiers et cognitifs permettant la dévalorisation et l'effacement des genres sociaux qui ne sont pas masculins.

Ex. Quand même, dit Fraine, une enfante toutes les deux années pendant quinze ou seize ans...

(Vonarburg $1999: 282$ )

\section{Ex. bien qu'elle ait fâcheusement tendance à amalgamer animales et humaines}

(La Baronne 2007 : 14)

\section{Ex. Le jeune Neutre qui était passé avant luiel avait été prévoyant}

(Rozenfeld 2014, chap.1 - 2/6)

Ex Al fait nuit dans le gymnase.

(Alpheratz $2015: 20$ )

\section{Ex. J'ai résistée.}

(D. $2016: 15)$

Ces évolutions et mouvements - politiques, technologiques, philosophiques, littéraires ayant influencé les modalités et les contenus discursifs en libérant la créativité d'une partie des francophones sur le plan du genre, nous paraissent à l'origine d'une " communauté épistémique ", au sens où Foucault l'envisage à partir du concept d'épistémè dans Les Mots et les choses (1966), c'est-à-dire une communauté reliée par un savoir propre à une époque, et au sens où ce concept est actuellement utilisé en philosophie politique, soit « les canaux par lesquels de nouvelles idées circulent des sociétés vers les gouvernements, et d'un pays à l'autre. » (Bossy \& Evrard 2010). 
Car le phénomène de l'inclusivité de genre dans les langues n'est pas inhérent à la France, mais à plusieurs pays occidentaux, notamment aux USA, en Suède et en Espagne, comme en témoigne la création récente ou la réactivation d'unités de genre neutre (ou commun ou inclusif) dans les langues de ces pays, et comme l'atteste son fait le plus célèbre : l'intégration du pronom personnel sujet hen dans le dictionnaire de l'Académie royale de Suède en 2015 .

De cette communauté épistémique fondée sur la conscience que le langage est constitutif de tout savoir et que la "vérité » est inhérente à qui l'édicte, sont nés les usages qui nous ont permis d'élaborer ce concept qu'est le français inclusif.

Enfin, cette variété du français standard est également liée à la reconnaissance politique à l'échelle nationale et internationale des personnes dites «non binaires » et qui regroupe les personnes «transgenres, transsexuelles, intersexes » ou «intersexuées » (Conseil de l'Europe 2017) en proposant un début de réponse à la question suivante : «Quels pronoms, quels titres, quels accords seraient possibles en français pour l'expression de la personne de sexe neutre?».

Ce terme de « genre social non binaire » étant d'une part un terme sociologique, d'autre part un concept en émergence et dans lequel le genre se scinde en une multiplicité de souscatégories (par ex. demi-fille, demi-garçon, deux esprits, neutrois, etc.), nous ne pouvons en dire que ce que nous en comprenons : être de genre non binaire, c'est avoir une identité de genre qui récuse le binarisme ou idéologie classant les individus selon une catégorisation binaire et exclusive des sexes et des genres.

Trois évènements éditoriaux participent également à la reconnaissance du français inclusif : la maison d'édition Hatier (Paris) a publié le premier manuel d'histoire destiné à des élèves d'école primaire (classe de CE2) en français inclusif (François \& Le Callennec 2017) et en 2018, outre notre Grammaire du français inclusif, est publié le Manuel de grammaire non sexiste et inclusive écrit par des juristes du Québec (Lessart \& Zaccour, 2018).

C'est dans cette séquence historique et cette communauté épistémique particulières que se situe notre observatoire.

\section{Termes métalinguistiques}

\subsection{Variation diaéthique}

Les productions langagières qui relèvent du français inclusif sont issues non seulement de sources textuelles diverses (guides de rédaction, courriels, essais, magazines, profils facebook, blogs, sites, romans) mais également d'un large prisme sociétal : institutions, presse, recherche universitaire, entreprise, associations, littérature, profils individuels sur les réseaux sociaux (Alpheratz 2018). Nous avons dit que le point commun à tous ces groupes et individus était une conscience de l'isomorphie pouvant exister entre genre social et grammatical. 
Cette conscience peut se manifester comme une glose mentale du locutaire qui, avant d'énoncer son discours, en choisit soigneusement les termes selon une variation qui relève non de l'espace, de la catégorie sociale ou du style, mais de la conscience de genre. $\mathrm{Al}^{\mathrm{v}}$ fait ainsi preuve d'une activité cognitivo-discursive qui nous semble suivre les phases suivantes (dans cet ordre) :

$1 /$ souci du mot juste et du respect des identités de genre, refus de la hiérarchisation entre les représentations symboliques et sociales instituée par l'emploi générique du genre grammatical masculin ;

2/conscience d'effectuer un acte politique motivée par l'espoir d'avoir une influence par ses choix linguistiques ;

3/évaluation de l'unité lexicale ou grammaticale possible à l'aune du principe d'inclusivité ;

4/jugement sur les paramètres linguistiques et extralinguistiques de l'unité examinée ;

5/créativité néologique et/ou commentaire épilinguistique si le français standard ne présente pas d'unité recevable.

Cette variation ne pouvait être qualifiée de «diatopique» (selon le critère géographique), ni de «diastratique» (selon le critère social et/ou démographique, le locutorat ${ }^{\mathrm{vi}}$ employant le français inclusif étant trop varié pour constituer un seul et unique groupe social), ni de «diaphasique » (selon le critère stylistique), quoique toutes ces variations puissent jouer un rôle dans le recours ou non au français inclusif. Car elle relève d'autre chose: d'un acte réfléchi, soit d'une politique d'inclusivité animée par une conscience de genre, conscience fondée sur le souci de l'expression et du respect des représentations symboliques et des catégories sociales minorisées. Cette variation nous semble donc pouvoir être qualifiée de " diaéthique ", mot formé par nous-même à partir du

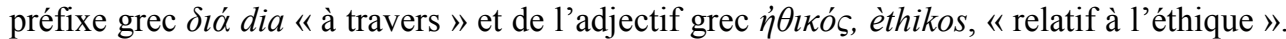
Sa définition peut être une variation relevant de la conscience de genre, d'identité, d'égalité et de la performativité du langage.

Cette variation diaéthique, qui pousse le locutorat à recourir au français inclusif, va à l'inverse de ce que Gaston Zink nomme « l'indifférence au rationnel » dans sa description du système des pronoms personnels du moyen français (1997). Le court extrait que nous en donnons, issu de sa conclusion, décrit cette indifférence comme étant l'une des conditions qui ont présidé à la généralisation du pronom masculin sujet $i l(z)$ à des référents féminins, ainsi que l'essor, au $\mathrm{XVI}^{\mathrm{e}}$ siècle, d'une tendance contraire à cette généralisation dans l'expression de la personne, du moins dans le cadre strict de l'expression du sujet.

« A ce trait de diversité s'ajoute celui, tout aussi caractéristique, d'indifférence au rationnel. Les sujets parlants ne tiennent pas pour absolument contraignantes les concordances de genre, de nombre ni même de personne dans les opérations de rappel. On étend « il (z) » à la référence féminine, en gardant les accords de « elle(s) ». [...] En 1500, la poussée de « il(z) » féminin semblait irrésistible $[\ldots]$ Or le $\mathrm{XVI}^{\mathrm{e}}$ siècle a déjoué l'attente en rejetant « il $(\mathrm{z})$ sont belles [...]». (Zink $1997: 383)$

$\mathrm{Au} \mathrm{XXI} \mathrm{e}^{\mathrm{e}}$ siècle, en ajoutant de nouveaux pronoms au système pronominal déjà en place (par ex. al, ul, iel, ille, celleux, etc.) afin de lever les ambigüités de communication sur la question du genre, le locutorat du français inclusif ne fait peut-être pas autre chose que trouver de nouveaux signes pour exprimer des concepts n'ayant jamais pu s'exprimer en langue. 
Alors que Moignet voit dans le pronom une catégorie vidée de toute matière notionnelle, parlant de « soustraction de substance notionnelle », ce locutorat semble au contraire « réinjecter » de la substance notionnelle dans les pronoms, selon sa perception analogique ou motivée de ces signes, dans l'expression de la personne. Ainsi, ces nouveaux pronoms sont peut-être " l'indice d'une meilleure concevabilité » pour reprendre l'expression de Moignet (1965), c'est-à-dire non seulement des variétés dans le discours, mais également l'indice d'un changement de système à l'œuvre dans la langue, qui procèderait d'une autre conceptualisation de la personne et de son expression.

Le système pronominal français a levé les ambiguïtés de communication du latin classique pour exprimer, par de nouveaux signes, un nouveau système de pensée. Ces nouveaux pronoms s'inscrivent peut-être dans la même tendance, qui nous semble avant tout psychique.

«Il est aussi évident que le système des pronoms personnels du français moderne est différent de celui du latin classique. Dans ce domaine, comme dans bien d'autres, la révolution romane a consisté à substituer un système de pensée à un autre, et conséquemment, à inventer un système sémiologique adéquat au système innové. » (Moignet $1965: 5$ )

\subsection{Epicénisation}

Les mots épicènes, de l'adjectif latin epicoenus, d'après le grec epikoinos " commun » (Neveu 2004: 120) sont des mots qui peuvent avoir plusieurs genres, et dont la morphologie ne varie pas en genre. Par exemple, l'adjectif apte peut qualifier un substantif de n'importe quel genre, le substantif journaliste peut avoir n'importe quel genre.

Notons que cette définition du terme épicène n'est pas la même que celle qui lui est donnée au Québec ou en Suisse dans le langage courant. Dans ces pays, épicène est un hyperonyme qui contient tous les processus langagiers permettant d'éviter le recours à l'emploi générique du masculin. Par exemple, le guide de rédaction Avoir bon genre à l'écrit : guide de rédaction épicène des Publications du Québec (Guénette et VachonL'heureux 2006) rassemble des processus dont la plupart n'ont rien à voir avec la définition du terme métalinguistique épicène. Pour ce guide, «les étudiants et les étudiantes » relève de la rédaction épicène alors que ni étudiants ni étudiantes ne sont des unités épicènes.

Cette différence entre la définition métalinguistique du terme et son usage dans ce type d'ouvrages à vocation didactique est, immanquablement, source de confusion et complique la tâche du linguiste. Par conséquent, nous nous en tiendrons au métalangage et ne doterons pas le terme épicène d'un caractère hyperonyme qu'il ne possède pas selon la définition de Neveu dans son Dictionnaire des sciences du langage (2004).

Le recours à des mots épicènes qui existent déjà et à la création de nouveaux épicènes pour éviter l'emploi générique du genre masculin nous semble relever d'un processus langagier que nous nommons «épicénisation». Il peut se définir comme suit : processus relevant du français inclusif qui, pour éviter l'emploi générique du genre masculin, consiste soit à avoir recours à un mot épicène (ex. élève plutôt qu'étudiant), soit à créer un mot épicène (ex. professionnèle), soit à rendre épicène un mot ayant déjà un genre (ex. une membre). 
Ex. L'emploi de termes épicènes comme membre (...) élève c'est-à-dire de termes non marqués du point de vue du genre grammatical, fait aussi partie des grands principes de l'écriture inclusive.

(Collège doctoral, site de l'Association des Doctorant.e.s de Paris-Sorbonne, doctoratp4.hypotheses.org, 01/07/2017)

Ex. la forme innovatrice " professionnèle » est substituée dans ce texte au dédoublement " professionnel » et " professionnelle "

(Labrosse $2002: 9$ )

Mais l'épicénisation crée une difficulté syntaxique en français inclusif. En effet, même si les substantifs épicènes ne fléchissent pas en genre, ils peuvent être amenés à déterminer le genre d'autres mots, donc à provoquer le fléchissement de ces derniers en genre, et à faire revenir le genre masculin (en emploi générique) que le locutorat du français inclusif s'emploie à éviter.

Ex. Tous les membres de l'association sont invités.

Ex. A partir de quinze minutes de retard, plus aucun élève n'aura accès à la salle d'examen.

Ex. Les plus grands professionnèles du secteur de l'édition se déclarent préoccupés par ce problème.

Ce problème peut être résolu par l'« écriture inclusive » ou « double flexion partielle ».

\subsection{Ecriture inclusive ou double flexion partielle}

«L'écriture inclusive » ou «double flexion partielle» est un processus langagier consistant à visibiliser la coprésence des genres sociaux dans l'écriture en ajoutant une marque morphologique de genre autre que celle du masculin à un mot déjà fléchi à ce genre et utilisé en emploi générique. L'écriture inclusive signale cette marque par l'insertion de signes ou de formats typographiques (point, point médian, tiret, parenthèses, barre oblique, capitale).

Notons que nous distinguons l'écriture inclusive ou « double flexion partielle » (par ex. les chanteur.euse.s) à la « double flexion totale » qui reprend le mot entier fléchi à un autre genre que le masculin (par ex. les chanteurs et les chanteuses) au lieu de reprendre uniquement les marques morphologiques de la flexion.

Ex. Tou.te.s les membres de l'association sont invité.e.s. (Point.)

Ex. A partir de quinze minutes de retard, plus aucun'e élève n'aura accès à la salle d'examen. (Point médian.)

Ex. Les plus grand-e-s professionnèles du secteur de l'édition se déclarent préoccupé$\boldsymbol{e}$-s par ce problème. (Tiret.)

Ex. Bonsoir à tou(te)s (Parenthèses.)

(Claire Grover, courriel sur liste EELV, 01/01/2017)

Ex. Les subalternes, peuvent-elles/ils (parler) être écouté-e-s? (Barre oblique)

(Groupe de recherche FIRA-MSH Paris Nord, UFRGS/Brésil et l'Université Nationale de Colombie, 12/01/2017) 
Ex. UniEs par l'écologie (Capitale.)

(Michel Sourrouille, courriel sur liste EELV, 05/01/2017)

Notons à nouveau une confusion entre la définition de ce terme de métalinguistique et son emploi dans l'usage courant, qui rassemble sous le terme "écriture inclusive » l'ensemble des processus langagiers du français inclusif. L'écriture inclusive n'est qu'un processus langagier du français inclusif, qui en comprend au moins dix autres (Alpheratz 2018). Cet usage dans le langage courant est le résultat d'une "extension sémantique », c'est-à-dire l'emploi d'un hyponyme au sens de son hyperonyme », pour reprendre Thibault (2018). C'est comme dire « langue » (hyponyme) à la place de « langage » (hyperonyme).

Si l'écriture inclusive résout le problème des mots employés au masculin générique lorsqu'ils sont déterminés par des substantifs épicènes, elle présente un problème d'économie linguistique en ce qu'elle emploie davantage d'unités (marques morphologiques et signes typographiques) que le genre masculin en emploi générique, qui est plus fonctionnel.

Nous pouvons également nous demander si, sur un plan sémiologique, la représentation de catégories minorisées par une marque morphologique et non un mot entier n'est pas problématique. Nous pouvons aussi reprocher à la double flexion partielle de ne pas atteindre totalement son objectif d'inclusivité en exprimant des référents de genre social non binaire avec des marques morphologiques binaires (c'est-à-dire exclusivement masculines et féminines). Bref, dans le premier cas, la double flexion partielle représente une catégorie minorisée (les femmes) par une marque morphologique au lieu d'un mot, et dans le deuxième cas, elle invisibilise une catégorie minorisée (les personnes de genre social non binaire) par une absence de marque morphologique.

Un autre processus langagier du français inclusif atteint peut-être plus efficacement l'objectif d'inclusivité que la double flexion partielle en réussissant à éviter les inconvénients qu'elle présente : c'est «l'hyperonymisation de genre ».

\subsection{Hyperonymisation de genre}

Le français inclusif se caractérise par plusieurs processus langagiers qui ont pour objectif de supprimer l'hyperonymie de genre ou caractère générique des mots de genre masculin. Pour continuer à désigner un ensemble de personnes de plusieurs genres, le locutorat de cette variété du français standard attribue cette hyperonymie à un autre mot ou groupe de mots.

Nous nommons « hyperonymisation de genre » le processus langagier qui consiste à préférer un terme qui ne peut être fléchi qu'à un seul genre à un terme qui peut être fléchi à plusieurs genres mais qui n'est utilisé qu'au masculin en emploi générique. Si besoin, l'hyperonymie de genre attribue cette propriété à ce nouveau mot (ou syntagme) si celui-ci ne la possède pas déjà.

Exemple 1. nous préférons désormais (...) des mots " englobants " (le corps professoral, le peuple, le public)

(Slate.fr, «Ecriture inclusive : Slate.fr choisit l'accord de proximité », 07/11/2017)

Pour reconnaître un mot hyperonyme (ou générique) de son mot hyponyme (inclus dans ce sur-ensemble qu'est l'hyperonyme), nous pouvons appliquer la procédure logique suivante : 
« L'identification d'un hyperonyme et d'un hyponyme peut être réalisée par une procédure logique très simple (...) : être A [hyponyme, ex. un basset], c'est être B [hyperonyme, ex. un chien], mais être B, ce n'est pas forcément être A. » (Neveu $2004: 151$ )

La relation d'un hyponyme à son hyperonyme est donc de l'ordre de l'inclusion stricte. Cette relation mathématique dite de "l'inclusion stricte " et symbolisée par le signe $\subset$ désigne une relation d'ordre entre ensembles selon laquelle l'hyponyme est un ensemble inclus dans l'hyperonyme, son sur-ensemble, sans être égal à lui. Dans l'exemple ci-dessus, nous pouvons traduire cette hyperonymisation de genre de la façon suivante :

Les professeurs $\subset$ le corps professoral

Les citoyens $\subset$ le peuple

Les lecteurs $\subset$ le public

Ces formules signifient que les ensembles désignés par les mots professeurs, citoyens et lecteurs sont respectivement inclus dans les ensembles désignés par les mots corps professoral, peuple et public, mais ne sont pas égaux à eux. Autrement dit, les mots choisis, corps professoral, peuple, public sont hyperonymes de professeurs, citoyens, lecteurs qui, en tant qu'hyponymes, ne peuvent désigner l'ensemble des personnes qui enseignent, qui font partie de la société française, qui lisent les articles de Slate.fr.

L'hyperonymisation de genre implique parfois une recatégorisation sémantique :

Exemple 2. Le premier tour de l'élection présidentielle 2017 est une élection primaire opposant toutes les Candidatures politiques (...) La Candidature qui rassemble le plus au premier tour aura gagné le premier tour et le second

(David Niconor, courriel sur liste EELV, 22/02/2017)

\section{candidats $\subset$ Candidature}

Cette formule signifie que l'ensemble désigné par le mot candidats est inclus dans l'ensemble désigné par le mot Candidature, mais n'est pas égal à lui. Le mot candidature se voit attribué de la propriété d'hyperonymie de genre, qu'il ne possédait pas, à la différence de corps professoral, peuple et public.

Cet exemple illustre également un cas de recatégorisation sémantique. Candidature perd son sens initial : le mot ne désigne plus l'état d'une personne candidate, ni l'action qui consiste à poser ou annoncer sa candidature, mais l'ensemble des personnes candidates tous genres confondus, en acquérant la propriété sémantique d'hyperonymie de genre. Selon le locuteur, un candidat (hyponyme) peut être représenté par une Candidature (hyperonyme). Le mot acquiert une capacité sémantique d'agentivité qu'il n'avait pas auparavant.

Parce que le mot acquiert un sens qu'il n'avait pas jusque-là, cette recatégorisation sémantique peut être source d'ambigüités. C'est d'ailleurs pour cette raison que le locuteur a recours à une figure de style, l'antonomase, pratiquée sur le mot candidature, pour expliciter son hyperonymisation.

Exemple 3. les Maisons Départementales des Personnes Handicapées (MDPH) sont chargées de l'accueil et de l'accompagnement des personnes handicapées et de leurs proches 
(Site des MDPH/Accueil)

\section{Des handicapés $\subset$ des personnes handicapées}

Cette formule signifie que l'ensemble désigné par le mot handicapés est inclus dans l'ensemble désigné par le syntagme nominal personnes handicapées, mais n'est pas égal à lui. Cet exemple, où le syntagme personnes handicapées est préféré au mot masculin en emploi générique handicapés illustre le cas d'hyperonymisation de genre dans lequel le mot hyperonyme choisi personne, possède déjà cette propriété : « Être humain, en particulier lorsqu'on ne peut ou ne veut préciser l'âge, le sexe, (...) l'apparence, (...) etc. » (Robert 2017 : 1870, à l'entrée " personne »). Un handicapé est une personne handicapée mais une personne handicapée n'est pas forcément un handicapé.

Dans cet exemple précis, (comme pour le corps professoral remplaçant les professeurs) nous notons le manque d'économie linguistique que présente la solution trouvée par le français inclusif pour remplacer le mot au masculin en emploi générique, lequel exprime le même sens avec moins d'unités : handicapés vs personnes handicapées (ou les professeurs vs le corps professoral).

Ces trois exemples montrent que l'hyperonymisation de genre n'implique pas l'évitement systématique du genre masculin mais uniquement l'emploi générique des mots qui peuvent être fléchis à plusieurs genres et qui sont employés au masculin. Ainsi, les mots choisis pour remplacer les mots de genre masculin en emploi générique sont parfois euxmêmes masculins : corps, peuple, public.

Ce nouveau terme de métalangue, hyperonymisation de genre, pose un problème à l'analyse du discours : comment s'assurer qu'un énoncé relève bien de ce procédé et de la variation diaéthique (conscience de genre, d'identité, d'égalité, de la performativité du langage), ou s'il ne relève pas seulement, par exemple, de la variation diaphasique ? Ce qui consisterait, dans le cadre de notre dernier exemple, à utiliser la première fois handicapés puis personnes handicapées, non par rejet d'une hiérarchie entre les représentations sociales associées aux genres grammaticaux, mais simplement pour éviter une répétition.

Pour l'instant, en tant que phénomène émergent, le français inclusif apparaît souvent dans des discours militants qui reprennent les sujets qui l'ont vu naître : mise à égalité des représentations symboliques et sociales associées aux genres grammaticaux et débats linguistiques sur ses modalités. Ces situations d'énonciation permettent d'identifier clairement ses différents processus (épicénisation, hyperonymisation, double flexion totale ou partielle, réactivation, etc.). Mais si le français inclusif se répand dans les usages et sort de ce cadre idéologique, l'identification d'une variation diaéthique risque peut-être de devenir moins évidente, notamment pour sa possible confusion avec la variation diaphasique.

\section{Genre neutre, commun ou inclusif}

Le genre grammatical neutre, qui aurait des marques morphologiques spécifiques, n'existe pas en français standard, seul existe un genre masculin dit « neutre ». Par exemple, il pleut ; c'est beau ; coursive non fumeur ; bus sans voyageur. Dans ces énoncés, les unités $i l$, beau, fumeur, voyageur sont fléchies au genre masculin sur le plan morphologique, tout en représentant une situation de neutralisation de genre sur le plan sémantique. Ici, neutre ne désigne pas un genre grammatical mais une valeur sémantique neutre attribuée à un mot fléchi au genre masculin. 
Mais si le genre neutre n'existe pas, alors de quel genre sont les mots ci-dessous ?

Ex. : Amigxs activistes d'Amérique latine... ce message est pour vous

(Profil facebook de Clémence Zamora-Cruz, 29/08/2017)

Ex. : Une manif pour touz' et pour tout le monde

(Régis, 2016)

Ex. : Beaucoup de réflexions ont notamment été menées avec l'autrim de ce blog. (uniqueensongenre.eklablog.fr/petit-dico-de-francais-neutre-inclusif, 26/01/2016)

Ex. Les rédacteurices. Qu'iels soit old school avec un carnet et un crayon à papier ou féru'e's du clavier, les mots restent leurs meilleurs alliés pour vous faire partager leurs rencontres, savoirs et coups de coeur.

(simonæ.fr/L'Equipe)

La situation d'énonciation (discours militant écrit par des locutaires pro français inclusif et parfois de genre social non binaire) nous permet d'identifier ces mots comme relevant de la variation diaéthique et d'interpréter les morphèmes dont ces unités sont pourvues ( $g x$ dans amigxs, $z$ ' dans touz', rim dans autrim) comme des signes morphologiques d'une flexion à un genre qui n'est ni masculin ni féminin.

Dans certains cas, les unités désignent des référents ni masculins ni féminins (par exemple certaines personnes de genre social non binaire), mais dans d'autres cas, les unités peuvent désigner des référents à la fois masculins et féminins (c'est le cas des groupes dont le genre des personnes est inconnu).

Nous voyons dans la dénomination « genre neutre » plutôt que « genre commun » ou « genre inclusif » plusieurs avantages.

L'adjectif latin neuter (masculin), neutra (féminin), neutrum (neutre) signifie « ni l'un ni l'autre » (cnrtl.fr/definition/neutre). Le terme neutre, en français moderne, comprend également ce sens. A priori, il serait donc pertinent pour qualifier un genre grammatical qui exclut le masculin et le féminin.

Par ailleurs, neutre présente une polysémie que ne présente pas le terme commun. Dans son acception contemporaine, neutre a plusieurs sens : " dans un état de neutralité ", " qui s'abstient de prendre parti », " qui appartient à une catégorie grammaticale où ne se manifeste pas le contenu mâle/femelle, la forme masculin/féminin » (Robert 2017 : 16871688).

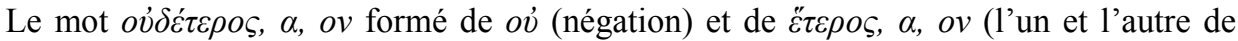
deux), tel qu'il est défini par Bailly dans son dictionnaire grec-français, recouvre l'ensemble des sens de notre définition : "d'aucun des deux genres » (Bailly, $1950: 1421$ ) si nous lui ajoutons entre parenthèses « exclusivement».

En effet, dire qu'une unité est de genre grammatical neutre au sens de «(exclusivement) d'aucun des deux genres ", c'est pouvoir dire non seulement qu'elle peut être des deux genres mais pas exclusivement, donc commune, mais également qu'elle peut être tout ce que le mot neutre implique, c'est-à-dire sans genre, ou de genre indifférent, ou inconnu, ce que ne peut pas désigner l'adjectif commun. Ce champ sémantique du mot neutre recouvre précisément les cinq sous-catégories de ce troisième genre grammatical, comme décrits ciaprès. 
Le genre neutre a l'avantage de réactiver un terme et une catégorie utilisée par les plus anciennes grammaires (Denys le Thrace cité par Lallot 1989: 44) avec le même sens que celui signalé par Bailly.

La dénomination «genre inclusif » peut également s'envisager. Les futures recherches linguistiques qui viendront sur le sujet nous aideront sans doute à trancher.

Par souci de rigueur, nous définirons donc le genre grammatical neutre comme suit : le genre grammatical neutre est une catégorie grammaticale associant des mots possédant des marques morphologiques ni de genre masculin ni de genre féminin, des mots possédant les marques de plusieurs genres ou des mots épicènes (identiques à tous les genres), à un sujet en structure impersonnelle, ou à un référent agenre, de genre social commun, inconnu, ou non binaire.

\subsection{Genre neutre, sous-catégorie « structure impersonnelle »}

Ex. Al ne manquerait plus que ça.

(Alpheratz 2015, p.543)

En structure impersonnelle, le sujet ne renvoie pas à un référent animé. Dans cette souscatégorie du genre neutre, la question du genre du « référent» est donc neutralisée, terme qui active deux sèmes du mot neutre /indétermination du genre/ (Robert 2017) et /ni masculin ni féminin/ (Bailly 1950, Lallot 1989).

\subsection{Genre neutre, sous-catégorie « agenre »}

Ex. Nous le traduirons par iel et nous emploierons ce pronom également pour les personnes agenres

(Well Well Well $3: 2$ )

Cette sous-catégorie permet de classer les énoncés exprimant des animés agenres. L'absence de genre de ces référents permet d'envisager le qualificatif de neutre au sens où, lorsqu'une question ne se pose pas, elle est neutralisée. Nous pouvons classer dans le genre neutre, sous-catégorie "agenre », les unités dont la morphologie ne relève pas d'une flexion de genre masculin ni féminin, et qui désignent des enfants, comme dans ce livre suédois utilisant le pronom personnel de genre neutre hen pour désigner le personnage principal, un enfant nommé Kivi.

Ex. «Oh non, surtout pas de chien! » Crie Kivi effrayæ.

Puis al continue: "Chère famille aimée :

Les chiens, entendez bien, ne sont pas si malins.

(Johansson, Lundqvist et Svensson, 2012, traduction de Julia Lindholm, 2017).

\subsection{Genre neutre, sous-catégorie " genre commun »}

Ex. Les rédacteurices. Qu'iels soit old school avec un carnet et un crayon à papier ou féru'e-s du clavier, les mots restent leurs meilleurs alliés pour vous faire partager leurs rencontres, savoirs et coups de coeur.

(simonæ.fr/L'Equipe) 
Cette sous-catégorie du genre neutre permet de classer les énoncés exprimant un référent ou des groupes de référents de plusieurs genres ou de tous les genres. Ce caractère commun, qui n'est pas exprimé par le sème /ni masculin ni féminin/ du terme neutre, l'est en revanche par la définition étendue de Bailly que nous proposons : (exclusivement) $n i$ masculin ni féminin qui permet premièrement de désigner des référents à la fois masculins et féminins mais pas exclusivement (d'un troisième genre social ?), deuxièmement de désigner des groupes de référents de divers genres.

\title{
2.4 Genre neutre, sous-catégorie « genre inconnu »
}

\author{
Ex. Une manif pour touz' et pour tout le monde \\ (Régis, 2016)
}

\section{Ex. Amigxs activistes d'Amérique latine... ce message est pour vous}

(Profil facebook de Clémence Zamora-Cruz, 29/08/2017)

Nous classons ces deux exemples dans le genre neutre, sous-catégorie "genre inconnu », car les termes tout le monde et d'Amérique latine, nous laissent supposer que le genre des référents faisant partie des groupes désignés, étant donné le caractère innombrable de leurs éléments, ne peut être connu des énonciatrices.

Cette sous-catégorie du genre neutre permet de classer des énoncés désignant des référents de genre inconnu. Or, inconnu ne signifie pas commun. Nous pouvons parfaitement imaginer que ces groupes innombrables ne soient pas composés de tous les genres.

Neutre nous semble donc plus approprié que commun pour désigner un genre grammatical « qui s'abstient de prendre parti » (Robert 2017) et où la question du genre est une fois encore « dans un état de neutralité » (Robert 2017).

\subsection{Genre neutre, sous-catégorie « genre non binaire »}

Ex. Beaucoup de réflexions ont notamment été menées avec l'autrim de ce blog.

(UESG, Blog Unique en son genre/Accueil, 26/01/2016)

Cette sous-catégorie du genre neutre permet de classer les énoncés exprimant des référents de genre social non binaire. Une personne se disant de genre non binaire pouvant être «d'aucun genre, d'aucun des deux genres» ou «des deux genres» (non exclusivement), «fluide », « neutrois », etc. classer les énoncés qui les désigne nous semble pertinent dans un genre neutre dont la polysémie est maximale en comparaison avec commun ou inclusif.

Dans notre typologie, nous choisissons délibérément de faire de la sous-catégorie « non binaire » une sous-catégorie du genre grammatical neutre du même niveau classificatoire que ses autres sous-catégories pour la raison suivante : le français inclusif étant fondé sur le rejet d'une hiérarchie entre les représentations symboliques et sociales qui sont associées aux genres grammaticaux, un troisième genre grammatical en français ne devrait pas pouvoir perpétuer cette hiérarchie en enlevant les spécificités grammaticales du genre masculin, utilisée pour classer les énoncés désignant une catégorie sociale et une seule (les hommes) pour les transférer à un autre genre, utilisé pour classer les énoncés désignant une catégorie sociale et une seule (les référents de genre social non binaire). Le genre neutre sert à classer des énoncés désignant parfois plusieurs catégories sociales sans que, 
morphologiquement, nous puissions voir un isomorphisme entre hiérarchie grammaticale et hiérarchie sociale, puisque les référents de genre social non binaire ne constituent pas une catégorie sociale dominante.

La sous-catégorie d'unités de genre grammatical neutre servant à désigner des référents de genre social non binaire est un hyponyme inclus dans l'hyperonyme qu'est le genre grammatical neutre, mais n'est pas égal à lui. Même si le genre grammatical neutre et sa sous-catégorie, le genre grammatical non binaire, peuvent partager les mêmes unités lexicales et structurales, le fait de les distinguer par une différence de nom et de niveau dans la catégorisation linguistique nous semble pouvoir permettre de lever toute ambigüité.

Nous voyons qu'en remplaçant l'hyperonymie du genre masculin par l'hyperonymie d'un genre neutre, le français inclusif n'abolit pas la hiérarchie dans la grammaire, mais seulement dans l'ontogénèse, sans reproduire la disposition et la désignation classificatoires qui caractérisent le masculin, et qui permettent un isomorphisme entre genre grammatical et genre social.

Notre conceptualisation $\mathrm{du}$ français inclusif et du genre neutre, en associant explicitement un genre grammatical à des genres sociaux (agenre, commun, inconnu, non binaire), donc en créant une analogie entre le signifiant et le signifié, propose peut-être une rupture dans l'histoire de l'interprétation du genre grammatical en langue française, interprétation basée (en partie) sur le caractère arbitraire du signe, c'est-à-dire de la relation signifiant/signifié. Cette conceptualisation « réinjecte » de la « substance notionnelle » pour reprendre le terme de Moignet, ou de la «motivation linguistique » selon les termes de la linguistique analogique, là où, jusqu'à présent, l'interprétation par les grammaires de la bicatégorisation du genre en français relayait la théorie saussurienne, en cela critiquée par les travaux sur les rapports isomorphes entre genre grammatical et genre social que nous avons cités.

\section{Perspectives et expérimentation}

L'état des lieux des unités et structures du français inclusif en 2017 donne à voir des unités manquantes, en concurrence, ou qui ne sont pas organisées en système, des recours qui augmentent le coût cognitif à la lecture, des propositions qui perdent en économie de moyens. Daniel Elmiger, qui synthétise quelques-unes des solutions de remplacement du genre masculin en emploi générique dans son article «Binarité du genre grammatical binarité des écritures ?»(2017), conclut sur la rareté des revendications théoriques ayant des applications pratiques et prédit un bel avenir à l'expérimentation.

Cette expérimentation du genre neutre est déjà effectuée en littérature (Rozenfeld 2014, Alpheratz 2018), en tenant compte des contraintes que cet art peut se donner (esthétiques, stylistiques, fonctionnelles). Mais pourquoi la littérature s'intéresse-t-elle plus particulièrement à cette ressource du français inclusif ? Peut-être parce que cette catégorie grammaticale permet de résoudre les problèmes de fonctionnalité et d'économie que posent ses autres ressources (voir exemple ci-après).

Cette expérimentation est aussi tentée hors littérature, en confrontant les individus ou la recherche qui la mènent à toutes les situations d'énonciation (cadre privé/professionnel, oral/écrit, contexte universitaire, littéraire, familial, sexuel, introspectif). C'est son avantage par rapport à une expérimentation limitée à un cadre artistique ou en laboratoire : multiplier les contextes et nous exposer à tous les imprévus de la vie. Ces expériences in situ sont aussitôt productrices d'effets et soumises notamment à la sanction immédiate de la 
nécessaire communicabilité du langage : certains néologismes, par exemple, sont aussitôt compris de l'allocutaire étant donné leur proximité phonétique avec des unités au masculin en emploi générique, d'autres suscitent une demande d'explication, d'autres enfin, peuvent aller jusqu'à provoquer une rupture de l'interaction verbale.

Cette expérimentation nous a notamment permis de proposer des solutions aux problèmes que pose cette variété du français standard, tel celui qu'évoque Viennot :

« La Fayette est sans conteste l'un des meilleurs

auteurs de son temps. » Comment dirons-nous donc

?»(Viennot $2014: 110)$

Ici, Viennot s'interroge sur l'alternative à trouver à auteurs (ainsi que les mots qui lui sont déterminés), unité fléchie au masculin en emploi générique et désignant l'ensemble des personnes de tous genres créant un ouvrage artistique ou scientifique. En l'occurrence, cet ensemble comprend le sujet de la première phrase, Marie-Madeleine Pioche de la Vergne, Comtesse de La Fayette (1634-1693), autrice du roman La Princesse de Clèves et désignée ici par La Fayette.

Notre proposition : La Fayette est sans conteste l'an ${ }^{\text {vii }}$ des meilleurz autaires de son temps.

Nous nous appuyons sur plusieurs logiques pour la formation des unités de genre neutre meilleurz et autaires.

Premièrement, nous nous inspirons du modèle lexicographique de Michel (2016) de mise en équation, c'est-à-dire à égalité, des unités fléchies en genre. Mais nous nous en écartons par une réelle mise au même niveau classificatoire des marques morphologiques propres à chaque genre. Nous ne reprenons pas la thèse de la «marque zéro » qui serait propre au genre masculin, et nous nous appuyons sur une conception agenre du mot, laquelle construit toutes les flexions en genre à partir d'une base délivrée de toute marque de genre, donc qui ne se confond plus avec le mot fléchi au masculin. Ici, les bases ne sont plus meilleur- (à laquelle s'ajoute l'affixe flexionnel $e$ au féminin) ni auteur (où le suffixe dérivationnel eur peut varier en rice au féminin), mais meill- (à laquelle s'ajoute le suffixe déjà fléchi au genre masculin eur) et aut- (à laquelle s'ajoute le suffixe déjà fléchi au genre masculin eur, ce qui ouvrirait peut-être la perspective d'analyser ce suffixe dérivationnel eur comme un affixe flexionnel de genre masculin).

$$
\text { Meill }\left\{\begin{array} { l } 
{ \text { eurs } } \\
{ \text { eures } } \\
{ \text { eurz } }
\end{array} \quad \text { aut } \left\{\begin{array}{c}
\text { eurs } \\
\text { rices } \\
\text { aires }
\end{array}\right.\right.
$$

Ce nouveau modèle inspiré de celui de Michel (2016) permet de «penser » le mot à partir de son radical, et non à partir du mot fléchi au genre masculin. Il révèle le traitement androcentrique du mot par les dictionnaires et les grammaires qui le mentionnent toujours en priorité sous sa forme masculine.

Deuxièmement, nous nous appuyons sur nos propositions de marques morphologiques spécifiques au genre neutre élaborées en fonction de l'observation des régularités présentées par les unités fléchies aux genres masculin et féminin.

Pour meilleur/meilleure/meilleurx (meilleurz au pluriel) la flexion au genre neutre se forme en s'appuyant sur la régularité vocalique et consantique en [œь] constatée dans la 
flexion binaire, régularité qu'elle reproduit par adjonction à la base du mot d'une variation en eurx, qui peut se prononcer [œь] avec un $x$ muet car la prononciation de cette consonne $\mathrm{x}$ en syllabe finale (rare en français) n'est pas validée par notre expérimentation (incompréhension systématique de l'allocutaire). Le pluriel se forme par la réactivation d'un morphème de pluriel $z$ en usage en ancien français (Labrosse 1996) sans que ce $z$ ne se prononce pour la même raison que $x$, par ex. meilleurz, inférieurz, mineurz.

Nous pouvons également envisager une harmonisation des régularités morphologiques observées dans la flexion en genre des unités en eur/eure, eur/euse et eur/ice qui pourraient n'avoir qu'une flexion en -aire au genre neutre, par ex. meilleur/meilleure/meillaire, chercheur/chercheuse/cherchaire, tuteur/tutrice/tutaire. Cette proposition de suffixation en aire, proche à l'oral du mot au genre masculin, présente l'avantage de ne pas créer d'incompréhension systématique chez l'allocutaire. La solution du genre neutre au problème posé par Viennot serait alors :

\section{La Fayette est sans conteste l'an des meillaires autaires de son temps.}

Pour auteur/autrice/autaire, la formation d'une flexion au genre neutre s'appuie sur l'opposition vocalique et consonantique constatée dans la flexion binaire en [œ]/[i] et en $[\mathrm{b}] /[\mathrm{s}]$ et reprend le son $[\mathrm{b}]$ présent dans la dernière syllabe des unités masculines et féminines par adjonction à la base du mot d'une troisième variation en aire qui peut se prononcer $[\varepsilon \varsigma]$. La flexion au pluriel se fait par adjonction de l'affixe flexionnel $s$ : par ex. des autaires, des sénataires, des piscicultaires.

Cette expérimentation linguistique, dont le cadre scientifique est défini précisément dans notre Grammaire (2018) permet non seulement de «trouver» et proposer des solutions aux problèmes que posent les autres processus langagiers du français inclusif, grâce à la multiplicité des situations d'énonciation où nous l'exerçons, mais permet également de trouver les instances de réfutation et de validation de la thèse d'un genre neutre, éléments essentiels qui garantissent la scientificité de notre démarche. Ainsi, les unités de genre neutre que nous proposons peuvent présenter des problèmes d'homonymie avec des unités du français standard, c'est le cas de l'article indéfini de genre neutre an avec le substantif de genre masculin an (sachant que de nombreuses homonymies existent en français, sans pour autant que l'ambigüité pose un problème à leur usage, par ex. tu bois/le bois, mineur (adjectif) mineur (substantif), etc.).

\section{CONCLUSION}

$\mathrm{Au} \mathrm{XXI}^{\mathrm{e}}$ siècle, les usages d'une communauté épistémique ayant analysé l'isomorphisme existant entre langage et pouvoir, et qui ne suivent pas les régularités du traitement du genre grammatical en français, obligent la recherche linguistique à définir les nouveaux concepts qu'ils mettent en œuvre, et à concevoir de nouveaux termes métalinguistiques pour analyser la variété qui les rassemble : le français inclusif.

Ainsi, la variation diaéthique, l'épicénisation, l'écriture inclusive ou double flexion partielle, l'hyperonymisation de genre et le genre neutre nous paraissent être autant d'outils permettant au linguiste d'objectiver ces nouveaux usages.

Si la discrimination logique et la hiérarchie entre classes et sous-classes n'ont pas été abolies en français inclusif, elles peuvent être redéfinies selon des modalités qui cherchent à 
rester circonscrites au sein de la catégorisation, c'est-à-dire non pas sur le plan du sens, mais sur le plan des structures.

L'expérimentation scientifique de tous les procédés langagiers du français inclusif et du genre neutre peuvent fournir des instances autant de validation que de falsifiabilité de la thèse de cette variété.

Par ailleurs, si une catégorie grammaticale aussi ancienne que le genre neutre se maintient depuis si longtemps, et si aujourd'hui elle semble pertinente pour classer de nouvelles productions langagières et leur permettre de sortir du " possible matériel » (Milner 1989) pour entrer dans la grammaticalité, c'est peut-être parce qu'elle vient combler un manque en français standard, pour exprimer les nouveaux concepts que sont l'inclusivité et les identités de genre. Parce qu'elles sont issues d'un locutorat très divers, les productions langagières du français inclusif constituent peut-être un nouveau système sémiologique servant à exprimer une nouvelle conceptualisation du monde, tout comme le système des articles et des pronoms en français l'avaient fait avant lui.

Reste que le français inclusif s'avère encore lacunaire pour atteindre une totale inclusivité. C'est qu'une langue française véritablement inclusive doit peut-être commander, non des solutions éparses et qui se concurrencent, mais une axiomatisation de ces nouveaux traitements du genre grammatical. Un travail à double volet morphosyntaxique et théorique pourrait peut-être atteindre l'utopie que le français inclusif ne fait pour l'instant que pointer du doigt : une langue sans hiérarchie entre les représentations symboliques et sociales qui sont associées aux genres grammaticaux, tant le caractère hautement flexionnel en genre de notre langue (en comparaison à l'anglais, par exemple) fait que l'emploi générique du genre masculin en affecte de nombreuses composantes.

\section{Références bibliographiques}

ALPHERATZ. (2015). Requiem. Charleston SC (USA) : Createspace.

(2018). Grammaire du français inclusif, Chateauroux : Vent solars.

ALTHUSSER, L. (1976), Positions : 1964-1975, 67-125, Paris : Les Éditions sociales.

AUSTIN, J. (1962). Quand dire c'est faire. Paris : Editions du Seuil.

BAILLY, A. (1950), Dictionnaire grec-français, Paris : Hachette.

BARONNE, La (2007), À la gloire de Pollo, Bouloc : Radio-Radio écritures.

BARTHES, R. (1971), Sade, Loyola, Fourier, Paris : Éditions du Seuil.

BECQUER, A., CERQUIGLINI, B. \& CHOLEWKA, N. (et. al.). 1999, Femme, j'écris ton nom, Guide d'aide à la féminisation des noms de métiers, titres, grades et fonctions, Paris : La Documentation française.

BENVENISTE, E. (1966). Problèmes de linguistique générale, t.1. Paris : Gallimard.

BHABHA, H. (2007). Les lieux de la culture. Une théorie postcoloniale, trad. de The Location of Culture par Françoise Bouillot. Paris : Payot.

BOSSY T. \& EVRARD A. (2010). "Communauté épistémique ». Dictionnaire des politiques publiques. Paris: Presses de Sciences Po.

BUTLER, J. (1990), Trouble dans le genre, trad. Cynthia Kraus, Paris : Editions La Découverte (2004), Défaire le genre, trad. de Undoing Gender par Maxime Cervulle (2012). Paris : Editions Amsterdam.

CONSEIL DE L'EUROPE. (2017). Promouvoir les droits humains et éliminer les discriminations à l'égard des personnes, Résolution n.2191. URL : http://assembly.coe.int/nw/xml/XRef/XrefXML2HTML-en.asp?fileid=24232\&lang=fr

D., T. (2016), Contes à rebours, Charleston SC (USA) : Createspace.

DE CARVALHO, P. (2010). «La Fin d'une illusion, ou l' « impersonnel » réduit et ramené à moi ». L'Impersonnel : la personne, le verbe, la voix, du partage des fonctions et de leur sémantisme dans 
les structures impersonnelles, Daviet-Taylor F. \& Bottineau D. (dir.), pp.53-71. Rennes : Presses Universitaires de Rennes.

DELPHY, C. (1975), «Un Féminisme matérialiste est possible », Nouvelles Questions Féministes 4, 1982, 50-86, Lausanne : Editions Antipodes.

DERRIDA, J. (1967), De la grammatologie, Paris : Editions de Minuit.

DRESSLER, W. \& KILANI SCHOCH, M. (2014). «L'Iconicité dans la morphologie flexionnelle du français. Le Français moderne, Revue de linguistique française, t.1. Paris : Éditions CILF.

ELMIGER, D. (2017). " Binarité du genre grammatical - binarité des écritures ? 》. Mots, les langages du politique, n.113, pp.37-52. URL : https://www.cairn.info/revue-mots-2017-1-page37.htm

FISCHER, O. \& NÄNNY M. (1999). Form Miming Meaning: Iconicity in Language and Literature, Amsterdam/Philadelphia : Benjamins.

FOUCAULT, M. (1966), Les Mots et les choses, Paris : Gallimard. (1975), Surveiller et punir, Paris : Gallimard.

FRANÇOIS, E. \& LE CALLENNEC, S. (2017), Questionner le monde-CE2, Cycle 2, Paris : Hatier GENETTE, G. (1966-1972), Figures II, Paris : Éditions du Seuil.

GODEFROY, F. (1982). Dictionnaire de l'ancienne langue française et de tous ses dialectes du IXe au XVe siècle, vol.3. (1891-1962). Genève : Slatkine. URL : http://gallica.bnf.fr/ark:/12148/bpt6k41891/f3.image

GUENETTE L. \& VACHON-L'HEUREUX P. (2006), Avoir bon genre à l'écrit : Guide de rédaction épicène, Office québécois de la langue française, Québec : Les Publications du Québec.

GUILLEMIN, C. (2016). Sexe, race et pratique du pouvoir. L'Idée de nature, (1978). Paris : iXe Editions.

HCE, Haut Conseil à l'Egalité entre Femmes et Hommes. (2016). Pour une communication publique sans stéréotypes de sexe, guide pratique. Paris : La Documentation française.

HERITIER, F. (2012). Masculin/Féminin I, La pensée de la différence, (1996). Paris : Odile Jacob.

HEROUT, R. (2014). Penser le possible de langue, en linguistique et en Poésie. URL https://www.shs-conferences.org/articles/shsconf/pdf/2014/05/shsconf_cmlf14_01347.pdf

JACOBSON, R. (1956). Fundamentals of language. The Hague : Mounton \& Co. (1965). «A la recherche de l'essence du langage ». Diogène, $n^{\circ} 51$. Paris : Gallimard.

JOHANSSON, B., LUNDQVIST J. \& SVENSSON E., (2012), Kivi \& Monsterhund, Stockholm: Olika Förlag.

KHAZNADAR, E. (1989). "Le Masculin premier ». Cahiers de grammaire, n.14, pp. 51-63. Toulouse : Université de Toulouse-Le Mirail ERSS-CNRS.

KOSOFSKY SEDGWICK, E. (1990). Epistémologie du placard, trad. de Epistemology of the Closet par Maxime Cervulle (2008). Paris : Editions Amsterdam.

LALLOT, J. (1998), La Grammaire de Denys le Thrace, 2e édition, Paris : CNRS-Éditions.

LA BARONNE. (2007). A la Gloire de Pollo. Toulouse : Radio-écritures.

LABROSSE, C. (1996). Pour une grammaire non sexiste. Montréal : Les Editions du remue-ménage. (2002). Pour une langue française non sexiste. Montréal : Les Editions des Intouchables.

LACAN, J. (1966). Ecrits I. Paris : Editions du Seuil.

LESSART, M. \& ZACCOUR, S. (2018). Manuel de grammaire non sexiste et inclusive. Le masculin ne l'emporte plus! Paris : Syllepse Editions.

MICHARD, C. (2012). " Rapport de sexage, effet idéologique et notion de sexe en français ». La Face cachée du genre, Chetcuti N \& Greco L. (dir.). Paris : Presses Sorbonne Nouvelle.

MICHEL, L. (2016). Thèse La Relation entre genre grammatical et dénomination de la personne en langue française (à paraître).

MILNER, J.-C. (1989), Introduction à une science du langage, Paris : Editions du Seuil (Points).

MOIGNET, G. (1965). Le Pronom personnel français, essai de psycho-systématique historique. Paris : Librairie C. Klincksieck.

(1973). Grammaire de l'ancien français. Paris : Klincksieck linguistiques.

(1981). Systématique de la langue française. Paris : Klincksieck.

MONNERET, P. (2011). "Motivation et analogie. Enjeux de la similarité en sciences du langage ». Philologia, n.56, pp.27-38. URL : https://hal.archives-ouvertes.fr/hal-01037900/document

NEEMAN, E. (2012). "Culture numérique et auctorialité : réflexions sur un bouleversement ». $A$ Contrario. URL : https://www.cairn.info/revue-a-contrario-2012-1.htm

NEVEU, F. (2004). Dictionnaire des sciences du langage. Paris : Armand Colin. 
NOBILE, L. (2014), «Introduction. Formes de l'iconicité ». Le Français moderne, 2014, n.1, tome 1. Paris : Éditions CILF.

ONISEP. (2014). L'Ecriture égalitaire : enjeux pour l'ONISEP et guide d'aide à la rédaction. URL : http://cache.media.education.gouv.fr/file/Egalite_F/G/95/8/Ecriture_egalitaire_a_1_Onisep_477958 .pdf

PROPP, V (1946), Les Racines historiques du conte merveilleux, trad. du russe par Lise Gruel-Apert (1983). Paris : Gallimard.

ROBERT, P. (2017). Dictionnaire Le Petit Robert, Rey A. \& Rey-Debove J. (dir.). Paris : Dictionnaires le Robert.

ROZENFELD, C. (2014). La Symphonie des abysses. Paris : Robert Laffont.

SAÏD, E. (1978), L'Orientalisme. L'Orient créé par l'Occident, trad. de Orientalism par Catherine Malamoud (1980). Paris : Editions du Seuil.

SCHMITT, E. (2015). «L'Ambiguïté épistémologique des big data : le cas de la donnée web en sciences sociales ». Big data, Open data, quelles valeurs ? Quels enjeux ? URL : https://www.academia.edu/29272069/Lambigu\%C3\%AFt\%C3\%A9_\%C3\%A9pist\%C3\%A9molo gique_des_big_data_le_cas_de_la_donn\%C3\%A9e_web_en_sciences_sociales

SEARLE, J. (1995). La Construction de la réalité sociale. Paris : Gallimard.

SERRES, M. (2011). " Petite poucette, la génération mutante - Entretien avec Michèle Nivelle ». Libération. URL : http://next.liberation.fr/culture/2011/09/03/petite-poucette-la-generationmutante 758710

THIBAULT, A. (2018), «Linguistique comparée des langues modernes. Semaine 3 : Les langues dans le temps, l'espace et la société (I) », André Thibault, URL : http://andre.thibault.pagespersoorange.fr/LingCompSemaine3.pdf

VIENNOT, E. (2014). Non, le masculin ne l'emporte pas sur le féminin ! Petite histoire des résistances de la langue française. Donnemarie-Dontilly : Éditions iXe.

VONARBURG, E. (1999), Chroniques du pays des mères, Québec : Éditions ALIRE inc. \& Élizabeth Vonarburg.

WHORF, B. (1956), Linguistique et anthropologie. Paris : Denoël.

YAGUELLO, M. (1978). Les Mots et les femmes, essai d'approche socio-linguistique de la condition féminine. Paris : Editions Payot.

ZINK, G. (1997). Morphosyntaxe du pronom personnel (non réfléchi) en moyen français (XIVe-XVe siècles). Genève : Librairie Droz S.A. (1995). Morphologie du français médiéval. Paris : PUF.

\footnotetext{
${ }^{\mathrm{i}}$ Substantif de genre neutre, remplace « locuteur ».

iii Substantif de genre neutre, remplace « auteur».

iv Substantif de genre neutre, remplace « lecteur».

${ }^{v}$ Pronom personnel de genre neutre, remplace « il ».

${ }^{\mathrm{vi}}$ Ensemble des locutaires.

vii Article indéfini de genre neutre, remplace « un ».
}

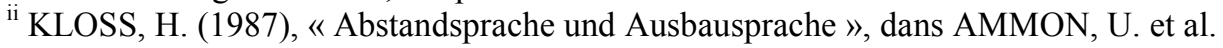
(éd.), Sociolinguistics / Soziolinguistik, vol. 1, Berlin/New York, de Gruyter, pp.302 - 8. En français : MULJAČIć, Zarko (1985), «L’enseignement de Heinz Kloss (modifications, implications, perspectives) », dans Langages, Paris, 21, n 83, pp. 53 - 63. 